\title{
On Cutwidth Parameterized by Vertex Cover
}

\author{
Marek Cygan • Daniel Lokshtanov • \\ Marcin Pilipczuk • Michał Pilipczuk • \\ Saket Saurabh
}

Received: 29 February 2012 / Accepted: 24 October 2012 / Published online: 8 November 2012

(C) The Author(s) 2012. This article is published with open access at Springerlink.com

\begin{abstract}
We study the CUTWIDTH problem, where the input is a graph $G$, and the objective is find a linear layout of the vertices that minimizes the maximum number of edges intersected by any vertical line inserted between two consecutive vertices. We give an algorithm for CUTWIDTH with running time $O\left(2^{k} n^{O(1)}\right)$. Here $k$ is the size of a minimum vertex cover of the input graph $G$, and $n$ is the number of vertices in $G$. Our algorithm gives an $O\left(2^{n / 2} n^{O(1)}\right)$ time algorithm for CUTWIDTH on bipartite graphs as a corollary. This is the first non-trivial exact exponential time algorithm for CUTWIDTH on a graph class where the problem remains NP-complete. Additionally, we show that CUTWIDTH parameterized by the size of the minimum vertex cover of the input graph does not admit a polynomial kernel unless NP $\subseteq$ coNP/poly. Our ker-
\end{abstract}

\footnotetext{
A preliminary version of this paper appeared at International Symposium on Parameterized and Exact Computation, IPEC 2011.

M. Cygan and M. Pilipczuk were supported by Polish Ministry of Science grant no. N206 567140 and Foundation for Polish Science. Michał Pilipczuk is supported by ERC grant "Rigorous Theory of Preprocessing", reference number 267959.
}

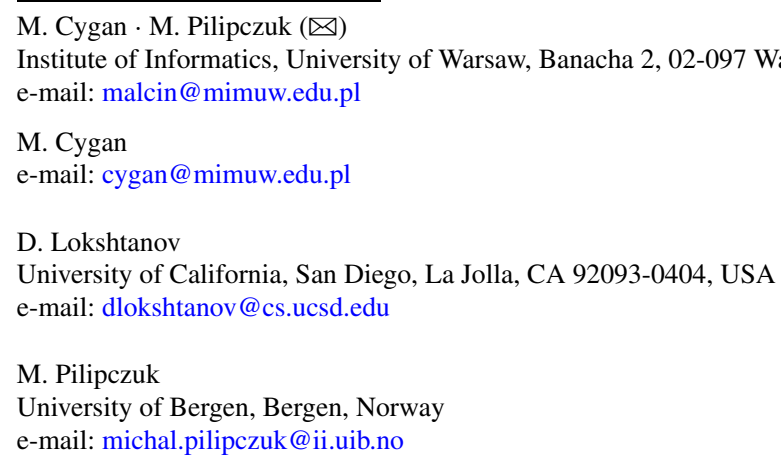

S. Saurabh

The Institute of Mathematical Sciences, Chennai 600113, India

e-mail: saket@imsc.res.in 
nelization lower bound contrasts with the recent results of Bodlaender et al. (ICALP, Springer, Berlin, 2011; SWAT, Springer, Berlin, 2012) that both TREEWIDTH and PATHWIDTH parameterized by vertex cover do admit polynomial kernels.

Keywords Cutwidth · Vertex cover parameterization · Parameterized complexity · Composition algorithms $\cdot$ Polynomial kernel

\section{Introduction}

In the CUTWIDTH problem we are given an $n$-vertex graph $G$ together with an integer $w$. The task is to determine whether there exists a linear layout of the vertices of $G$ such that any vertical line inserted between two consecutive vertices of the layout intersects with at most $w$ edges (see Sect. 2 for a formal definition). The cutwidth $(c w(G))$ of $G$ is the smallest $w$ for which such a layout exists. The problem has numerous applications [10, 23, 24, 29], ranging from circuit design [1, 27] to protein engineering [4]. Unfortunately CUTWIDTH is NP-complete [18], and remains so even when the input is restricted to subcubic planar bipartite graphs $[13,28]$ or split graphs where all independent set vertices have degree 2 [20]. On the other hand, the problem has a factor $O\left(\log ^{2}(n)\right)$-approximation on general graphs [26] and is polynomial time solvable on trees $[12,32]$, graphs of constant treewidth and constant degree [31], threshold graphs [20], proper interval graphs [34] and bipartite permutation graphs [19].

In this article we study the complexity of computing cutwidth exactly on general graphs, where the running time is measured in terms of the size of the smallest vertex cover of the input graph $G$. A vertex cover of $G$ is a vertex set $S$ such that every edge of $G$ has at least one endpoint in $S$. We show that CUTWIDTH can be solved in time $2^{k} n^{O(1)}$ where $k$ is the size of the smallest vertex cover of $G$. An immediate consequence of our algorithm is that CUTWIDTH can be solved in time $2^{n / 2} n^{O(1)}$ on bipartite graphs. This is the first non-trivial exact exponential time algorithm for CUTWIDTH on a graph class where the problem is NP-complete. Furthermore, our algorithm improves considerably over the previous best algorithm for CUTWIDTH parameterized by vertex cover [15], whose running time is $O\left(2^{2^{O(k)}} n^{O(1)}\right)$ (however, it was not the focus of [15] to optimize the running time dependence on $k$ ).

Additionally, we show that CUTWIDTH parameterized by vertex cover does not admit a polynomial kernel unless NP $\subseteq$ coNP/poly. A polynomial kernel for CUTWIDTH parameterized by vertex cover is a polynomial time algorithm that takes as input a CUTWIDTH instance $(G, w)$, where $G$ has a vertex cover of size at most $k$ and outputs an equivalent instance $\left(G^{\prime}, w^{\prime}\right)$ of CUTWIDTH such that $G^{\prime}$ has at most $k^{O(1)}$ vertices. We show that unless $\mathrm{NP} \subseteq$ coNP/poly such a kernelization algorithm can not exist. This contrasts with the recent results of Bodlaender et al. [7, 8] that both TREEWIDTH and PATHWIDTH parameterized by the vertex cover number of the input graph do admit polynomial size kernels.

Context of Our Work The CUTWIDTH problem is one of many graph layout problems, where the task is to find a permutation of the vertices of the input graph that 
optimizes a problem specific objective function. Graph layout problems, such as TREEWIDTH, BANDWIDTH and HAMILTONIAN PATH are not amenable to "branching" techniques, and hence the design of faster exact exponential time algorithms for these problems has resulted in several new and useful tools. For example, Karp's inclusion-exclusion based algorithm [25] for HAMILTONIAN PATH was the first application of inclusion-exclusion in exact algorithms. Another example is the introduction of potential maximal cliques as a tool for the computation of treewidth. Most graph layout problems (with the exception of BANDWIDTH) admit an $O\left(2^{n} n^{O(1)}\right)$ time dynamic programming algorithm $[2,21]$. For several of these problems, faster algorithms with running time below $O\left(2^{n}\right)$ have been found [3, 16, 30], a stellar example is the recent algorithm by Björklund [3] for HAMILTONiAn PATH. The CUTWIDTH problem is perhaps the best known graph layout problem for which a $O\left(2^{n} n^{O(1)}\right)$ time algorithm is known, yet no better algorithm has been found. Hence, whether such an improved algorithm exists is a tantalizing open problem. While we do not resolve this problem in this article, we make considerable progress; hard instances of CUTWIDTH cannot contain any independent set of size $c n$ for any $c>0$.

Our choice of the vertex cover number as a relevant parameter for the CUTWIDTH problem originates in a recent interest in structural parameters (see e.g. [6-8, 22]), in particular from the study of a very closely related problems of computing treewidth and pathwidth of the input graph. Note that both these problems can be easily seen to be AND-compositional when parameterized by the target treewidth or pathwidth of the graph (see [5] for discussion and relevant definitions) and an AND-composition, together with existence of a polynomial kernel, is now known to cause a collapse of the polynomial hierarchy [14]. This situation, together with the importance of the TREEWIDTH and PATHWIDTH problems, motivated Bodlaender et al. [7, 8] to investigate their other, stronger parameterizations. Among many other results, they have proven that both these problems admit a polynomial kernel with respect to the vertex cover of the graph, while such a kernel is unlikely if we parameterize by the deletion distance to a clique. We show that, although CUTWIDTH seems very similar to PATHWIDTH, these problems behave differently with respect to polynomial kernelization: CUTWIDTH does not admit a polynomial kernel when parameterized by the vertex cover number unless NP $\subseteq$ coNP/poly, which is known to imply a collapse of the polynomial hierarchy to its third level $[11,33]$.

Organization of the Paper In Sect. 2 we present a dynamic programming algorithm which computes cutwidth in time $O\left(2^{k} n^{O(1)}\right)$ for a given vertex cover of size $k$, whereas in Sect. 3 we show that CUTWIDTH parameterized by vertex cover does not admit a polynomial kernel unless NP $\subseteq$ coNP/poly. Section 4 is devoted to concluding remarks.

Notation All graphs in this paper are undirected and simple. For a vertex $v \in V$ we define its neighbourhood $N_{G}(v)=\{u: u v \in E(G)\}$ and closed neighbourhood $N_{G}[v]=N_{G}(v) \cup\{v\}$. If $G$ is clear from the context, we might omit the subscript. For $X \subseteq V$ we denote $N_{G}[X]=\bigcup_{v \in X} N_{G}(v) \backslash X$. 


\section{Faster Cutwidth Parameterized by Vertex Cover}

In this section we show that given a graph $G=(C \cup I, E)$ such that $C$ is a vertex cover of $G$ of size $k$, we can compute the cutwidth of $G$ in time $O\left(2^{k} n^{O(1)}\right)$, using a dynamic programming approach. We start by showing that there always exists an optimal ordering of a specific form.

For an ordering $\sigma=v_{1} \cdots v_{n}$ of $V=C \cup I$ we define $V_{i}=\left\{v_{j}: j \leq i\right\}$. For vertices $u$ and $v \in V$ we say that $u \leq_{\sigma} v$ if $u$ occurs before $v$ in $\sigma$. Denote by $\delta\left(V_{i}\right)$ the number of edges between $V_{i}$ and $V \backslash V_{i}$. The cutwidth of the ordering, $c w_{\sigma}(G)$, is defined as the maximum of $\delta\left(V_{i}\right)$ for $i=1,2, \ldots,|V|-1$, and the cutwidth of the graph $G$ is the minimum cutwidth over all possible orderings $\sigma$ of $V$. The rank of a vertex $v_{i}$ with respect to an ordering $\sigma$ is denoted by $\operatorname{rank}_{\sigma}\left(v_{i}\right)$ and it is equal to $\left|N\left(v_{i}\right) \backslash V_{i}\right|-\left|N\left(v_{i}\right) \cap V_{i}\right|$. Notice that $\delta\left(V_{i+1}\right)=\delta\left(V_{i}\right)+\operatorname{rank}_{\sigma}\left(v_{i+1}\right)$ and hence $\delta\left(V_{i}\right)=\sum_{j \leq i} \operatorname{rank}_{\sigma}\left(v_{j}\right)$. Moving a vertex $v_{p}$ backward to position $q$ with $q<p$ results in the ordering

$$
\sigma^{\prime}=v_{1} v_{2} \cdots v_{q-2} v_{q-1} \mathbf{v}_{\mathbf{p}} v_{q} v_{q+1} \cdots v_{p-2} v_{p-1} v_{p+1} v_{p+2} \cdots v_{n}
$$

Moving $v_{p}$ forward to a position $q$ with $q>p$ results in the ordering

$$
\sigma^{\prime}=v_{1} v_{2} \cdots v_{p-2} v_{p-1} v_{p+1} v_{p+2} \cdots v_{q-2} v_{q-1} \mathbf{v}_{\mathbf{p}} v_{q} v_{q+1} \cdots v_{n} .
$$

Notice that any vertex with odd degree must have (nonzero) odd rank. Moreover, moving a vertex backward cannot decrease its rank, whereas moving a vertex forward cannot increase its rank.

Lemma 1 If moving $v_{p}$ backward to position $q$ results in an ordering $\sigma^{\prime}$ such that $\operatorname{rank}_{\sigma^{\prime}}\left(v_{p}\right) \leq 0$ then $c w_{\sigma^{\prime}}(G) \leq c w_{\sigma}(G)$. If moving $v_{p}$ forward to position $q$ results in an ordering $\sigma^{\prime}$ such that $\operatorname{rank}_{\sigma^{\prime}}\left(v_{p}\right) \geq 0$ then $c w_{\sigma^{\prime}}(G) \leq c w_{\sigma}(G)$.

Proof Suppose moving $v_{p}$ backward to position $q$ results in an ordering $\sigma^{\prime}$ such that $\operatorname{rank}_{\sigma^{\prime}}\left(v_{p}\right) \leq 0$. For every non-negative integer $i$ define $V_{i}^{\prime}$ to contain the first $i$ vertices of $\sigma^{\prime}$. Then, for every $i<q$ and $i \geq p$ we have $V_{i}^{\prime}=V_{i}$ and hence $\delta\left(V_{i}^{\prime}\right)=$ $\delta\left(V_{i}\right)$. For every $i$ such that $q \leq i<p$ we have that $V_{i}^{\prime}=V_{i-1} \cup\left\{v_{p}\right\}$. Observe that for any other vertex $v_{j}, j \neq p, \operatorname{rank}_{\sigma^{\prime}}\left(v_{j}\right) \leq \operatorname{rank}_{\sigma}\left(v_{j}\right)$, while $\operatorname{rank}_{\sigma^{\prime}}\left(v_{p}\right) \leq 0$. Thus $\delta\left(V_{i}^{\prime}\right)=\operatorname{rank}_{\sigma^{\prime}}\left(v_{p}\right)+\sum_{j \leq i-1} \operatorname{rank}_{\sigma^{\prime}}\left(v_{j}\right) \leq \delta\left(V_{i-1}\right)$ and $c w_{\sigma^{\prime}}(G) \leq c w_{\sigma}(G)$. The proof that if moving $v_{p}$ forward to position $q$ results in an ordering $\sigma^{\prime}$ such that $\operatorname{rank}_{\sigma^{\prime}}\left(v_{p}\right) \geq 0$ then $c w_{\sigma^{\prime}}(G) \leq c w_{\sigma}(G)$ is analogous.

Lemma 1 allows us to rearrange optimal orderings. Let $\sigma$ be an optimal cutwidth ordering of $G, c_{1} c_{2} \cdots c_{k}$ be the ordering which $\sigma$ imposes on $C$ and $C_{i}=\left\{c_{1}, \ldots, c_{i}\right\}$ for every $i$. Observe that if $u$ and $v$ are both in $I$ then moving $u$ does not affect the rank of $v$. In particular, if moving $u$ yields the ordering $\sigma^{\prime}$, then $\operatorname{rank}_{\sigma^{\prime}}(v)=$ $\operatorname{rank}_{\sigma}(v)$. For every vertex $u \in I$ with odd degree and $\operatorname{rank}_{\sigma}(u)<0$ we move $u$ backward to the leftmost position where $u$ has rank -1 . For every vertex $u \in I$ with odd degree and $\operatorname{rank}_{\sigma}(u)>0$ we move $u$ forward to the rightmost position where $u$ has rank 1 . For every vertex of the set $I$ with even degree we move it (forward or 
Fig. 1 Illustration of the definition of the sets $X(S, v)$ and $Y(S, v)$

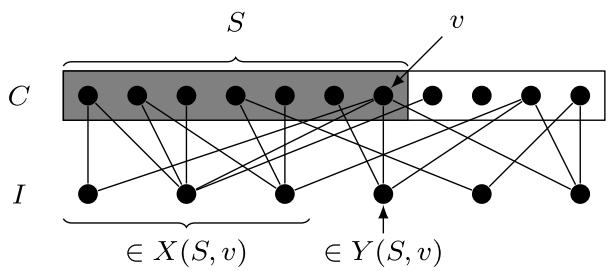

backward) to the rightmost position where $u$ has rank 0 . This results in an optimal cutwidth ordering $\sigma^{\prime}$ with the following properties.

1. For every vertex $v \in I$ of even degree $\operatorname{rank}_{\sigma^{\prime}}(v)=0$ and every vertex $v \in I$ of odd degree $\operatorname{rank}_{\sigma^{\prime}}(v) \in\{-1,1\}$.

2. For every vertex $v \in I$ such that $\operatorname{rank}_{\sigma^{\prime}}(v) \geq 0$ and $c_{i} \in C$ we have $c_{i} \leq \sigma^{\prime} v$ if and only if $\left|N(v) \cap C_{i}\right| \leq\left|N(v) \backslash C_{i}\right|$.

3. For every vertex $v \in I$ such that $\operatorname{rank}_{\sigma^{\prime}}(v)<0$ and $c_{i} \in C$ we have $c_{i} \leq \sigma^{\prime} v$ if and only if $\left|N(v) \cap C_{i-1}\right|<\left|N(v) \backslash C_{i-1}\right|$.

Define $I_{0}^{\prime}$ and $I_{k}^{\prime}$ to be the set of vertices in $I$ appearing before $c_{1}$ and after $c_{k}$ in $\sigma^{\prime}$, respectively. For $i$ between 1 and $k-1$ we denote $I_{i}^{\prime}$ the set of vertices in $I$ appearing between $c_{i}$ and $c_{i+1}$ in $\sigma^{\prime}$. For any $i$, if $I_{i}^{\prime}$ contains any vertices of rank -1 , we move them backward to the position right after $c_{i}$. This results in an ordering $\sigma^{\prime \prime}$ where for every $i$, all the vertices of $I_{i}^{\prime}$ with negative rank appear before all the vertices of $I_{i}^{\prime}$ with non-negative rank. By Lemma 1 and the fact that moving a vertex from independent set does not affect the rank of another vertex from the independent set we have that $\sigma^{\prime \prime}$ is still an optimal cutwidth ordering. Also, $\sigma^{\prime \prime}$ satisfies the properties $1-3$. We say that an ordering $\sigma$ is $C$-good if it satisfies properties $1-3$ and orders the vertices between vertices of $C$ in such a way that all vertices of negative rank appear before all vertices of non-negative rank. The construction of $\sigma^{\prime \prime}$ from an optimal ordering $\sigma$ proves the following lemma.

Lemma 2 Let $G=(C \cup I, E)$ be a graph and $C$ be a vertex cover of $G$. There exists an optimal cutwidth ordering $\sigma$ of $G$ which is $C$-good.

In a $C$-good ordering $\sigma$, consider a position $i$ such that $c_{i} \in C$. Because of the properties of a $C$-good ordering we can essentially deduce $V_{i} \cap I$ from $V_{i} \cap C$ and the vertex $c_{i}$. We will now formalize this idea. For a set $S \subseteq C$ and vertex $v \in S$ we define the set $X(S, v) \subseteq I$ as follows. A vertex $u \in I$ of even degree is in $X(S, v)$ if $|N(u) \cap S|>|N(u) \backslash S|$. A vertex $u \in I$ of odd degree is in $X(S, v)$ if $\mid N(u) \cap$ $(S \backslash\{v\})|>| N(u) \backslash(S \backslash\{v\}) \mid$. Now we define the set $Y(S, v)$. A vertex $u \in I$ is in $Y(S, v)$ if $u v \in E$ and $|(N(u) \backslash\{v\}) \cap S|=|(N(u) \backslash\{v\}) \backslash S|$. Note that the vertices in $Y(S, v)$ have odd degrees and $Y(S, v)$ is disjoint with $X(S, v)$. We refer to Fig. 1 for an illustration. The following observation follows directly from the properties of a $C$-good ordering.

Observation 3 In a $C$-good ordering $\sigma$ let $i$ be an integer such that $c_{i} \in C$ and let $S=V_{i} \cap C$. Then $X\left(S, c_{i}\right) \subseteq V_{i} \cap I \subseteq X\left(S, c_{i}\right) \cup Y\left(S, c_{i}\right)$. 
A prefix ordering $\phi$ is a set $V_{\phi} \subseteq C \cup I$ together with an ordering of $V_{\phi}$. The size of the prefix ordering $\phi$ is just $\left|V_{\phi}\right|$. Similarly to normal orderings we define $V_{i}^{\phi}=\left\{v_{1} \cdots v_{i}\right\}$. Let $c_{1} c_{2} \cdots c_{\left|V_{\phi} \cap C\right|}$ be the ordering imposed on $V_{\phi} \cap C$ by $\phi$, and for every $i \leq\left|V_{\phi} \cap C\right|$ we set $C_{i}^{\phi}=\left\{c_{1}, \ldots, c_{i}\right\}$. The rank of a vertex $v \in V_{\phi}$ with respect to $\phi$ is defined as $\operatorname{rank}_{\phi}\left(v_{i}\right)=\left|N\left(v_{i}\right) \backslash V_{i}^{\phi}\right|-\left|N\left(v_{i}\right) \cap V_{i}^{\phi}\right|$. We now extend the notion of being $C$-good from orderings of $G$ to prefix orderings of $G$ in such a way that the restriction of any $C$-good ordering $\sigma$ of $G$ to the first $t$ vertices, where $v_{t} \in C$, must be $C$-good. We say that a prefix ordering $\phi=v_{1} \cdots v_{t}$ of size $t$ with $v_{t} \in C$ is $C$-good if the following conditions are satisfied.

1. For every vertex $v \in V_{\phi} \cap I$ of even degree, $\operatorname{rank}_{\phi}(v)=0$ and for every vertex $v \in V_{\phi} \cap I$ of odd degree, $\operatorname{rank}_{\phi}(v) \in\{-1,1\}$.

2. $X\left(V_{\phi} \cap C, v_{i}\right) \subseteq V_{\phi} \cap I \subseteq X\left(V_{\phi} \cap C, v_{i}\right) \cup Y\left(V_{\phi} \cap C, v_{i}\right)$

3. For every vertex $v \in X\left(V_{\phi} \cap C, c_{i}\right)$ such that $\operatorname{rank}_{\phi}(v) \geq 0$ and $c_{i} \in V_{\phi} \cap C$ we have $c_{i} \leq_{\phi} v$ if and only if $\left|N(v) \cap C_{i}^{\phi}\right| \leq\left|N(v) \backslash C_{i}^{\phi}\right|$.

4. For every vertex $v \in X\left(V_{\phi} \cap C, c_{i}\right)$ such that $\operatorname{rank}_{\phi}(v)<0$ and $c_{i} \in V_{\phi} \cap C$ we have $c_{i} \leq_{\phi} v$ if and only if $\left|N(v) \cap C_{i-1}^{\phi}\right|<\left|N(v) \backslash C_{i-1}^{\phi}\right|$.

5. Between two vertices $c_{i}, c_{i+1} \in C \cap V_{\phi}$, all vertices with $\operatorname{rank}_{\phi}(v)<0$ come before all vertices with $\operatorname{rank}_{\phi}(v) \geq 0$.

Comparing the properties of $C$-good orderings and $C$-good prefix orderings it is easy to see that the following lemma holds.

Lemma 4 Let $\sigma=v_{1} \cdots v_{n}$ be a $C$-good ordering and let $\phi$ be the restriction of $\sigma$ to the first $t$ vertices, such that $v_{t} \in C$. Then $\phi$ is a $C$-good prefix ordering.

For a prefix ordering $\phi$ define the cutwidth of $G$ with respect to $\phi$ to be $c w_{\phi}(G)=$ $\max _{i \leq\left|V_{\phi}\right|} \delta\left(V_{i}^{\phi}\right)$. For a subset $S$ of $C$ and vertex $v \in S$, define $T(S, v)$ to be the minimum value of $c w_{\phi}(G)$ where the minimum is taken over all $C$-good prefix orderings $\phi$ with $V_{\phi} \cap C=S$ and $v$ being the last vertex of $\phi$. Notice that property 5 of $C$-good prefix orderings implies that in a $C$-good prefix ordering $\phi$ there must be some $i$ with $v_{i} \in C \cap V_{\phi}$ such that $c w_{\phi}(G)=\delta\left(V_{i}\right)$ or $c w_{\phi}(G)=\delta\left(V_{i-1}\right)$. Also, notice that for any set $S \subseteq C$ and vertices $u, v \in S$ we have that $X(S \backslash\{v\}, u) \subseteq X(S, v)$ and $Y(S \backslash\{v\}, u) \subseteq X(S, v)$. Finally, observe that for any set $S \subseteq C$ and vertex $v \in S$, every vertex $u \in Y(S, v)$ is adjacent to $v$ and satisfies $|(N(u) \backslash\{v\}) \cap S|=$ $|(N(u) \backslash\{v\}) \backslash S|$. Thus, for any set $I^{\prime} \subseteq I$ with $X(S, v) \subseteq I^{\prime} \subseteq X(S, v) \cup Y(S, v)$ the value of $\delta\left(S \cup I^{\prime}\right)$ depends only on $\left|Y(S, v) \cap I^{\prime}\right|$ and not on $Y(S, v) \cap I^{\prime}$ in general. We let $Y_{i}(S, v)$ be an arbitrary subset of $Y(S, v)$ of size $i$. The discussion above yields that the following recurrence holds for $T(S, v)$, where $S \subseteq C$ and $v \in S$.

$$
T(S, v)=\min _{u \in S} \min _{0 \leq i \leq|Y(S, v)|} \max \left\{\begin{array}{l}
\delta\left(S \cup X(S, v) \cup Y_{i}(S, v)\right) \\
\delta\left((S \backslash\{v\}) \cup X(S, v) \cup Y_{i}(S, v)\right) \\
T(S \backslash\{v\}, u)
\end{array}\right\} .
$$

Observe that $c w(G)=\min _{v \in C} T(S, v)$ because in any ordering $\sigma$ all vertices of $I$ appearing after the last vertex of $C$ must have negative rank. Thus the recurrence 
above naturally leads to a dynamic programming algorithm for CUTWIDTH running in time $O\left(2^{k} n^{O(1)}\right)$. This proves the main theorem of this section.

Theorem 5 There is an algorithm that given a graph $G=(C \cup I, E)$ such that $C$ is a vertex cover of $G$, computes the cutwidth of $G$ in running time $O\left(2^{|C|}(|C|+\right.$ $\left.|I|)^{O(1)}\right)$. Thus, MINIMUM CUTWIDTH on bipartite graphs can be solved in time $O\left(2^{n / 2} n^{O(1)}\right)$, where $n$ is the number of vertices of the input graph.

\section{Kernelization Lower Bound}

In this section we show that CUTWIDTH parameterized by vertex cover does not admit a polynomial kernel unless NP $\subseteq$ coNP/poly.

\subsection{The Auxiliary Problem}

We begin by introducing an auxiliary problem, namely HYPERGRAPH MINIMUM BISECTION. Let $H=(V, E)$ be a multihypergraph with $|V|=n$, where $n$ is even. A bisection of $V$ is a colouring $\mathcal{B}: V \rightarrow\{0,1\}$ such that $\left|\mathcal{B}^{-1}(0)\right|=\left|\mathcal{B}^{-1}(1)\right|=$ $n / 2$. For a hyperedge $e$ let us define the cost of $e$ with respect to a bisection $\mathcal{B}$ as $\operatorname{cost}(e, \mathcal{B})=\min \left(\left|e \cap \mathcal{B}^{-1}(0)\right|,\left|e \cap \mathcal{B}^{-1}(1)\right|\right)$. The cost of a bisection is defined as the sum of the contributions of the hyperedges, i.e., $\operatorname{cost}(\mathcal{B})=\sum_{e \in E} \operatorname{cost}(e, \mathcal{B})$.

HYPERGRAPH MINIMUM BISECTION

Parameter: $n$

Input: Multihypergraph $H$ with $n$ vertices, where $n$ is even; an integer $k$

Question: Does there exist a bisection of $H$ with cost at most $k$ ?

In the case when all the hyperedges are in fact edges (have cardinalities 2) and there are no multiedges, the problem is equivalent to the classical MINIMUM BISECTION problem. As Minimum BISECTION is NP-hard, HyPERGRAPH Minimum BISECTION is also NP-hard, so NP-complete as well.

The goal now is to prove that CUTWIDTH parameterized by the size of vertex cover does not admit a polynomial kernel, unless NP $\subseteq$ coNP/poly. We do it in two steps. First, using the OR-distillation technique of Bodlaender et al. [5] (with the backbone theorem proven by Fortnow and Santhanam [17]) we prove that HYPERGRAPH MINIMUM BISECTION does not admit a polynomial kernel, unless NP $\subseteq$ coNP/poly. Second, we present a parameterized reduction from HYPERGRAPH MINIMUM BISECTION to CUTWIDTH parameterized by vertex cover.

\subsection{No Polynomial Kernel for HyPergraph Minimum Bisection}

We use the OR-distillation technique (first introduced by Bodlaender et al. [5]) put into the framework called cross-composition [6]. Let us recall the crucial definitions.

Definition 6 (Polynomial Equivalence Relation [6]) An equivalence relation $\mathcal{R}$ on $\Sigma^{*}$ is called a polynomial equivalence relation if (1) there is an algorithm that given 
two strings $x, y \in \Sigma^{*}$ decides whether $\mathcal{R}(x, y)$ in $(|x|+|y|)^{O(1)}$ time; (2) for any finite set $S \subseteq \Sigma^{*}$ the equivalence relation $\mathcal{R}$ partitions the elements of $S$ into at most $\left(\max _{x \in S}|x|\right)^{O(1)}$ classes.

Definition 7 (Cross-Composition [6]) Let $L \subseteq \Sigma^{*}$ and let $Q \subseteq \Sigma^{*} \times \mathbb{N}$ be a parameterized problem. We say that $L$ cross-composes into $Q$ if there is a polynomial equivalence relation $\mathcal{R}$ and an algorithm which, given $t$ strings $x_{1}, x_{2}, \ldots, x_{t}$ belonging to the same equivalence class of $\mathcal{R}$, computes an instance $\left(x^{*}, k^{*}\right) \in \Sigma^{*} \times \mathbb{N}$ in time polynomial in $\sum_{i=1}^{t}\left|x_{i}\right|$ such that (1) $\left(x^{*}, k^{*}\right) \in Q$ iff $x_{i} \in L$ for some $1 \leq i \leq t$; (2) $k^{*}$ is bounded polynomially in $\max _{i=1}^{t}\left|x_{i}\right|+\log t$.

Theorem 8 [6, Theorem 9] If $L \subseteq \Sigma^{*}$ is NP-hard under Karp reductions and $L$ cross-composes into the parameterized problem $Q$ that has a polynomial kernel, then $\mathrm{NP} \subseteq$ coNP/poly.

Lemma 9 HyPERGRAPH MINIMUM BISECTION, parameterized by the size of the universe, does not admit a polynomial kernel, unless NP $\subseteq$ coNP/poly.

Proof As Minimum Bisection is NP-hard under Karp reductions, it suffices to prove that it cross-composes into the HYPERGRAPH MINIMUM BISECTION problem, parameterized by $n$ - the size of the universe. Let $R$ be an equivalence relation on $\Sigma^{*}$ defined as follows:

- all words that do not correspond to instances of Minimum BisECTION form one equivalence class;

- all the well-formed instances are partitioned into equivalence classes having the same number of vertices, the same number of edges and the same demanded cost of the bisection.

It is straightforward that $R$ is a polynomial equivalence relation. Therefore, we can assume that the composition algorithm is given a sequence of instances $\left(G_{0}, k\right),\left(G_{1}, k\right), \ldots,\left(G_{t-1}, k\right)$ of Minimum BISECTION with $\left|V\left(G_{i}\right)\right|=n$ and $\left|E\left(G_{i}\right)\right|=m$ for all $i=0,1, \ldots, t-1$ ( $n$ is even). Moreover, by copying some instances if necessary we can assume without losing generality that $t=2^{l}$ for some integer $l$. Note that in this manner we do not increase the order of $\log t$.

We now proceed to the construction of the composed HYPERGRAPH MINIMUM Bisection instance $(H, K)$. Let $N=2 m \cdot\left((l+2) 2^{l-1}-1\right)+2 k+1$ and $M=$ $N\left(l^{2}-l\right)+N$. We begin by creating two sets of vertices $A_{0}$ and $A_{1}$, each of size $2 n l$. We introduce each set $A_{0}, A_{1}$ as a hyperedge of the constructed hypergraph $M$ times.

Then, we introduce $2 l$ vertices $s_{i}^{0}, s_{i}^{1}$ for $i=0,1, \ldots, l-1$ and denote the set of all these vertices by $S$. For every $i<j$ we put $N$ times each hyperedge $\left\{s_{i}^{0}, s_{j}^{0}\right\}$, $\left\{s_{i}^{0}, s_{j}^{1}\right\},\left\{s_{i}^{1}, s_{j}^{0}\right\},\left\{s_{i}^{1}, s_{j}^{1}\right\}$. Thus, the hypergraph induced by $S$ is a clique without a matching, repeated $N$ times. Furthermore, for every $p=0,1$ and $i=0,1, \ldots, l-1$ we construct a set $S_{i}^{p}$ of $n-1$ vertices and put $S_{i}^{p} \cup\left\{s_{i}^{p}\right\}$ as a hyperedge of the constructed hypergraph $M$ times. 
Fig. 2 The constructed hypergraph $H$ for $l=3$; encircled vertices indicate the hyperedge $e^{0}$ constructed for $e=v_{1}^{5} v_{2}^{5} \in E\left(G_{5}\right)$
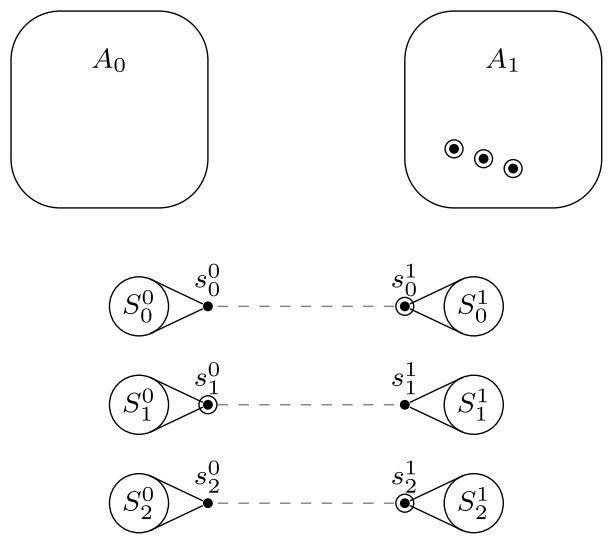

$W$

$\begin{array}{ll}v_{1} & v_{2} \\ \odot & \bigcirc\end{array}$

Now, we construct a set of $n$ vertices $v_{1}, v_{2}, \ldots, v_{n}$ and denote it by $W$. For every instance $G_{a}$ we arbitrarily choose an ordering of its vertices and denote it by $v_{1}^{a}, v_{2}^{a}, \ldots, v_{n}^{a}$. Let $b_{l-1} b_{l-2} \cdots b_{1} b_{0}$ be the binary representation of $a$, with trailing zeroes added so that its length is equal to $l$. For every edge $e=v_{g}^{a} v_{h}^{a} \in E\left(G_{a}\right)$ we create two hyperedges:

- $e^{0}$, consisting of vertices $v_{g}, v_{h}, s_{i}^{b_{i}}$ for all $i=0,1, \ldots, l-1$ and $l$ vertices from $A_{1}$, chosen arbitrarily;

$-e^{1}$, consisting of vertices $v_{g}, v_{h}, s_{i}^{1-b_{i}}$ for all $i=0,1, \ldots, l-1$ and $l$ vertices from $A_{0}$, chosen arbitrarily.

Finally, we set the expected cost of the bisection to $K=M-1=N\left(l^{2}-l\right)+2 m$. $\left((l+2) 2^{l-1}-1\right)+2 k$. We refer to Fig. 2 for an illustration.

Assume that some graph $G_{a}$ has a bisection $\mathcal{B}$ having cost at most $k$. Let $b_{l-1} b_{l-2} \cdots b_{1} b_{0}$ be the binary representation of $a$, as in the previous paragraph. We now construct a bisection $\mathcal{B}^{\prime}$ of $H$ as follows:

- for each $u \in A_{0}$ we set $\mathcal{B}^{\prime}(u)=0$, for each $u \in A_{1}$ we set $\mathcal{B}^{\prime}(u)=1$;

- for each $u \in S_{i}^{p} \cup\left\{s_{i}^{p}\right\}$ for $p=0,1, i=0,1, \ldots, l-1$ we set $\mathcal{B}^{\prime}(u)=p+b_{i}$ $(\bmod 2)$;

- for each $v_{j} \in W$ we set $\mathcal{B}^{\prime}\left(v_{j}\right)=\mathcal{B}\left(v_{j}^{a}\right)$.

Observe that $\mathcal{B}^{\prime}$ bisects each of the sets $A_{0} \cup A_{1}, S$ and $W$, so it is a bisection. We now prove that its cost is at most $K$. Let us count the contribution to the cost from every hyperedge of $H$.

Each copy of the hyperedges $A_{0}, A_{1}$ and $S_{i}^{p} \cup\left\{s_{i}^{p}\right\}$ for $p=0,1, i=0,1, \ldots, l-1$ has zero contribution, as it is monochromatic. The edges of $H[S]$ have contribution 0 or 1 , depending on whether the endpoints are coloured in the same or in a different way in $\mathcal{B}^{\prime}$. There are $l$ vertices $s_{i}^{p}$ that map to 0 in $\mathcal{B}^{\prime}$ and $l$ that map to 1 , so there are $l^{2}$ pairs of vertices coloured in a different way. Between every pair of vertices 
there are $N$ edges, apart from the pairs $\left(s_{i}^{0}, s_{i}^{1}\right)$. Note that all these pairs are coloured differently; therefore, there are exactly $N\left(l^{2}-l\right)$ edges in $H[S]$ contributing 1 to the cost.

Take $c \in\{0,1, \ldots, t-1\}$ such that $c \neq a$. Let $d_{l-1} d_{l-2} \cdots d_{0}$ be the binary representation of $c$. For $e \in E\left(G_{c}\right)$ let us count the contribution to $\operatorname{cost}\left(\mathcal{B}^{\prime}\right)$ of the hyperedges $e^{0}$ and $e^{1}$. Suppose that $q=\left|\left\{i: b_{i} \neq d_{i}\right\}\right|>0$. Among vertices of $e^{0}, l$ from $A_{1}$ are coloured 1, $q$ from $S$ are coloured 1 as well and $l-q$ from $S$ are coloured 0 . In total, we have $l+q$ vertices coloured 1 and $l-q$ coloured 0 , so regardless of the colouring of the remaining two vertices from $W$, the contribution is equal to the number of vertices coloured 0 in $e^{0}$, namely $l-q+\left|e^{0} \cap W \cap \mathcal{B}^{\prime-1}(0)\right|$. Analogously, the contribution of the hyperedge $e^{1}$ is equal to the number of vertices of $e^{1}$ coloured 1 , namely $l-q+\left|e^{1} \cap W \cap \mathcal{B}^{\prime-1}(1)\right|$. As there are exactly two vertices in $e^{0} \cap W=e^{1} \cap W, \operatorname{cost}\left(e^{0}, \mathcal{B}^{\prime}\right)+\operatorname{cost}\left(e^{1}, \mathcal{B}^{\prime}\right)=2(l-q)+2$. Thus, the total contribution of hyperedges $e^{0}, e^{1}$ for $e \in E\left(G_{c}\right)$ is equal to $2 m(l-q)+2 m$.

Now we count the contribution of the edges $e^{0}$ and $e^{1}$ for $e \in E\left(G_{a}\right)$. Analogously as in the previous paragraph, both edges $e^{0}, e^{1}$ contain $l$ vertices coloured $0, l$ vertices coloured 1 plus two vertices from $W$. If both these vertices are coloured in the same way, the sum of the contributions of $e^{0}$ and $e^{1}$ is equal to $2 l$; however, if the vertices are coloured differently, the sum is equal to $2 l+2$. As the cost of bisection $\mathcal{B}$ was at most $k$, the total contribution of edges $e^{0}, e^{1}$ for $e \in E\left(G_{a}\right)$ is at most $2 m l+2 k$.

Finally, we sum up the contributions:

$$
\begin{aligned}
\operatorname{cost}\left(\mathcal{B}^{\prime}\right) & \leq N\left(l^{2}-l\right)+2 m \sum_{q=1}^{l}(l-q+1)\left(\begin{array}{l}
l \\
q
\end{array}\right)+2 m l+2 k \\
& =N\left(l^{2}-l\right)+2 m \cdot\left(2^{l}-1\right)+2 m \sum_{q=0}^{l}(l-q)\left(\begin{array}{l}
l \\
q
\end{array}\right)+2 k \\
& =N\left(l^{2}-l\right)+2 m \cdot\left(2^{l}-1\right)+2 m l 2^{l-1}+2 k \\
& =N\left(l^{2}-l\right)+N-1=K .
\end{aligned}
$$

We proceed to the second direction. Assume that we have a bisection $\mathcal{B}^{\prime}$ of $H$ such that $\operatorname{cost}\left(\mathcal{B}^{\prime}\right) \leq K$. Observe that as $M>K$, both the sets $A_{0}, A_{1}$ are monochromatic with respect to $\mathcal{B}^{\prime}$. Moreover, they have to be coloured differently, as they contain more than half of the vertices of the graph in total. Without losing generality we can assume that $A_{0}$ is coloured in colour 0 , while $A_{1}$ is coloured in colour 1 , by flipping the colours if necessary.

Now consider the set $S_{p}^{i} \cup\left\{s_{p}^{i}\right\}$ for $p=0,1, i=0,1, \ldots, l-1$. Analogously as in the previous paragraph, $S_{p}^{i} \cup\left\{s_{p}^{i}\right\}$ has to be monochromatic. Furthermore, observe that exactly $l$ such sets have to be coloured 0 in $\mathcal{B}^{\prime}$ and the same number have to be coloured 1, as every set $S_{p}^{i} \cup\left\{s_{i}^{p}\right\}$ contains the same number of vertices as the set $W$ and $\mathcal{B}^{\prime}$ is a bisection. Therefore, $\mathcal{B}^{\prime}$ has to bisect each of the sets $A_{0} \cup A_{1}, S$ and $W$.

Exactly $l$ vertices $s_{i}^{p}$ are coloured 0 in $\mathcal{B}^{\prime}$ and exactly $l$ are coloured 1 . Let $r$ be the number of indices $i$, such that $s_{i}^{0}$ and $s_{i}^{1}$ are coloured differently. Observe that analogously to our previous arguments, the contribution of the edges of $H[S]$ to $\operatorname{cost}\left(\mathcal{B}^{\prime}\right)$ is equal to $N\left(l^{2}-r\right)=N\left(l^{2}-l\right)+N(l-r)$. If $r<l$, then $\operatorname{cost}\left(\mathcal{B}^{\prime}\right) \geq$ 
$N\left(l^{2}-l\right)+N>K$, a contradiction. Therefore, all the pairs $\left(s_{i}^{0}, s_{i}^{1}\right)$ are coloured differently.

Let $a$ be a number with binary representation $\mathcal{B}^{\prime}\left(s_{l-1}^{0}\right) \mathcal{B}^{\prime}\left(s_{l-2}^{1}\right) \cdots \mathcal{B}^{\prime}\left(s_{0}^{1}\right)$. Consider a bisection $\mathcal{B}$ of $G_{a}$ defined as follows: $\mathcal{B}\left(v_{i}^{a}\right)=\mathcal{B}^{\prime}\left(v_{i}\right)$. We claim that the cost of $\mathcal{B}$ is at most $k$. Indeed, the same computations as in the previous part of the proof show that

$$
\operatorname{cost}\left(\mathcal{B}^{\prime}\right)=N\left(l^{2}-l\right)+2 m\left((l+2) 2^{l-1}-1\right)+2 \operatorname{cost}(\mathcal{B})
$$

Therefore, as $\operatorname{cost}\left(\mathcal{B}^{\prime}\right) \leq K$, then $\operatorname{cost}(\mathcal{B}) \leq k$.

\subsection{From HypergRaph Minimum Bisection to CutwidTh}

Let us briefly recall the notion of polynomial parameter transformations.

Definition 10 [9] Let $P$ and $Q$ be parameterized problems. We say that $P$ is polynomial parameter reducible to $Q$, written $P \leq p Q$, if there exists a polynomial time computable function $f: \Sigma^{*} \times \mathbb{N} \rightarrow \Sigma^{*} \times \mathbb{N}$ and a polynomial $p$, such that for all $(x, k) \in \Sigma^{*} \times \mathbb{N}$ the following holds: $(x, k) \in P$ iff $\left(x^{\prime}, k^{\prime}\right)=f(x, k) \in Q$ and $k^{\prime} \leq p(k)$. The function $f$ is called a polynomial parameter transformation.

Theorem 11 [9] Let $P$ and $Q$ be parameterized problems and $\tilde{P}$ and $\tilde{Q}$ be the unparameterized versions of $P$ and $Q$ respectively. Suppose that $\tilde{P}$ is NP-hard and $\tilde{Q}$ is in NP. Assume there is a polynomial parameter transformation from $P$ to $Q$. Then if $Q$ admits a polynomial kernel, so does $P$.

We apply this notion to our case.

Lemma 12 There exists a polynomial-time algorithm that, given an instance of the HYPERGRAPH MINIMUM BISECTION problem with $n$ vertices, outputs an equivalent instance of the CUTWIDTH problem along with its vertex cover of size $n$.

Proof Let $(H=(V, E), k)$ be an instance of HyPERGRAPH Minimum Bisection given in the input, where $|V|=n$ ( $n$ is even) and $|E|=m$. We construct a graph $G$ as follows.

Let us denote $N=m n+1$. We begin by taking the whole set $V$ to be the set of vertices of $G$. For every distinct $u, v \in V$ we introduce $N$ new vertices $x_{u, v}^{i}$ for $i=1,2, \ldots, N$, each connected only to $u$ and $v$. Then, for every $e \in E$ we introduce a new vertex $y_{e}$ connected to all $v \in e$. Denote the set of all vertices $x_{u, v}^{i}$ by $X$ and the set of all vertices $y_{e}$ by $Y$. This concludes the construction. Observe that $V$ is a vertex cover of $G$ of size $n$. We now prove that $H$ has a bisection with cost at most $k$ if and only if $G$ has cutwidth at most $n^{2} N / 4+k$.

Assume that $H$ has a bisection $\mathcal{B}$ with cost at most $k$. Let us order the vertices of the graph $G$ as follows. First, we order the vertices from $V$ : we place $\mathcal{B}^{-1}(0)$ first, in any order, and then $\mathcal{B}^{-1}(1)$, in any order. Then, we place every $x_{u, v}^{i}$ anywhere between $u$ and $v$. At the end, for every $e \in E$ we place $y_{e}$ at the beginning if at least half of the vertices of $e$ are in $\mathcal{B}^{-1}(0)$, and in the end otherwise. Vertices $y_{e}$ at the beginning and at the end are arranged in any order. 
Now, we prove that the cutwidth of the constructed ordering is at most $n^{2} N / 4+k$. Consider any cut $C$, dividing the order on $V(G)$ into a first part $V_{1}$ and a second part $V_{2}$. Suppose that $\left|V_{1} \cap V\right|=n / 2-l$ for some $-n / 2 \leq l \leq n / 2$, thus $\left|V_{2} \cap V\right|=$ $n / 2+l$. Observe that $C$ cuts exactly $N(n / 2-l)(n / 2+l)=n^{2} N / 4-l^{2} N$ edges between $V$ and $X$. Note that there are not more than $n m<N$ edges between $V$ and $Y$. Therefore, if $l \neq 0$, then $C$ can cut at most $n^{2} N / 4-N+n m<n^{2} N / 4+k$ edges.

We are left with the case when $l=0$. Observe that $V_{1} \cap V=\mathcal{B}^{-1}(0)$ and $V_{2} \cap V=$ $\mathcal{B}^{-1}(1)$. Moreover, the cut $C$ cuts exactly $n^{2} N / 4$ edges between sets $V$ and $X$. As far as edges between $V$ and $Y$ are concerned, for every hyperedge $e \in E$, the cut $C$ cuts exactly $\operatorname{cost}(e, \mathcal{B})$ edges incident on $y_{e}$. As $\operatorname{cost}(\mathcal{B}) \leq k$, the cut $C$ cuts at most $n^{2} N / 4+k$ edges.

Now assume that there is an ordering of vertices of $G$ that has cutwidth at most $n^{2} N / 4+k$. We construct a bisection $\mathcal{B}$ of $H$ as follows. Let $\mathcal{B}(v)=0$ for every $v$ among the first $n / 2$ vertices from $V$ with respect to the ordering, and $\mathcal{B}(v)=1$ for $v$ among the second $n / 2$ vertices. We now prove that the cost of this bisection is at most $k$.

Let $C$ be any cut dividing the order into the first part $V_{1}$ and the second part $V_{2}$, such that $V_{1} \cap V=\mathcal{B}^{-1}(0)$ and $V_{2} \cap V=\mathcal{B}^{-1}(1)$. As the cutwidth of the ordering is at most $n^{2} N / 4+k, C$ cuts at most $n^{2} N / 4+k$ edges. Observe that $C$ needs to cut at least $n^{2} N / 4$ edges between sets $V$ and $X$, therefore it cuts at most $k$ edges between sets $V$ and $Y$. For every hyperedge $e \in E, C$ cuts at least $\operatorname{cost}(e, \mathcal{B})$ edges incident to $y_{e}$, thus $\operatorname{cost}(\mathcal{B}) \leq k$.

From Lemmas 9, 12 and Theorem 11 we conclude the following.

Theorem 13 CUTWIDTH parameterized by the size of vertex cover does not admit a polynomial kernel, unless $\mathrm{NP} \subseteq \mathrm{coNP} /$ poly.

\section{Conclusions}

In this paper we studied the complexity of computing the cutwidth of a graph parameterized by the size of a given vertex cover. We have shown an algorithm with running time $O\left(2^{k} n^{O(1)}\right)$, where $k$ is the cardinality of the vertex cover and $n$ is the number of vertices of the graph. Moreover, we have proven that polynomial kernelization of the problem is unlikely, thus counterpoising the recent result of Bodlaender et al. [7].

The thrilling and natural question is whether the insight we have given into the problem can be a starting point to breaking the $2^{n}$ barrier for an exact algorithm computing cutwidth. Our result implies that one can assume that in any hard instance all the independent sets are small, i.e., of size not larger than $c n$ for an arbitrarily small constant $c>0$.

Open Access This article is distributed under the terms of the Creative Commons Attribution License which permits any use, distribution, and reproduction in any medium, provided the original author(s) and the source are credited. 


\section{References}

1. Adolphson, D., Hu, T.C.: Optimal linear ordering. SIAM J. Appl. Math. 25, 403-423 (1973)

2. Bellman, R.: Dynamic programming treatment of the travelling salesman problem. J. ACM 9(1), 6163 (1962)

3. Björklund, A.: Determinant sums for undirected hamiltonicity. In: FOCS, pp. 173-182. IEEE Comput. Soc., Los Alamitos (2010)

4. Blin, G., Fertin, G., Hermelin, D., Vialette, S.: Fixed-parameter algorithms for protein similarity search under mRNA structure constraints. J. Discrete Algorithms 6, 618-626 (2008)

5. Bodlaender, H.L., Downey, R.G., Fellows, M.R., Hermelin, D.: On problems without polynomial kernels. J. Comput. Syst. Sci. 75(8), 423-434 (2009)

6. Bodlaender, H.L., Jansen, B.M.P., Kratsch, S.: Cross-composition: a new technique for kernelization lower bounds. In: Schwentick, T., Dürr, C. (eds.) STACS, LIPIcs, vol. 9, pp. 165-176 (2011). Schloss Dagstuhl-Leibniz-Zentrum fuer Informatik

7. Bodlaender, H.L., Jansen, B.M.P., Kratsch, S.: Preprocessing for treewidth: a combinatorial analysis through kernelization. In: Aceto, L., Henzinger, M., Sgall, J. (eds.) ICALP (1). Lecture Notes in Computer Science, vol. 6755, pp. 437-448. Springer, Berlin (2011)

8. Bodlaender, H.L., Jansen, B.M.P., Kratsch, S.: Kernel bounds for structural parameterizations of pathwidth. In: Fomin, F.V., Kaski, P. (eds.) SWAT. Lecture Notes in Computer Science, vol. 7357, pp. 352363. Springer, Berlin (2012)

9. Bodlaender, H.L., Thomassé, S., Yeo, A.: Kernel bounds for disjoint cycles and disjoint paths. Theor. Comput. Sci. 412(35), 4570-4578 (2011)

10. Botafogo, R.A.: Cluster analysis for hypertext systems. In: Korfhage, R., Rasmussen, E.M., Willett, P. (eds.) SIGIR, pp. 116-125. ACM, New York (1993)

11. Cai, J., Chakaravarthy, V.T., Hemaspaandra, L.A., Ogihara, M.: Competing provers yield improved Karp-Lipton collapse results. Inf. Comput. 198(1), 1-23 (2005)

12. Chung, M., Makedon, F., Sudborough, I., Turner, J.: Polynomial time algorithms for the min cut problem on degree restricted trees. SIAM J. Comput. 14, 158-177 (1985)

13. Diaz, J., Penrose, M., Petit, J., Serna, M.: Approximating layout problems on random geometric graphs. J. Algorithms 39, 78-117 (2001)

14. Drucker, A.: New limits to classical and quantum instance compression. In: FOCS. IEEE Comput. Soc., Los Alamitos (2012, to appear)

15. Fellows, M.R., Lokshtanov, D., Misra, N., Rosamond, F.A., Saurabh, S.: Graph layout problems parameterized by vertex cover. In: Hong, S.H., Nagamochi, H., Fukunaga, T. (eds.) ISAAC. Lecture Notes in Computer Science, vol. 5369, pp. 294-305. Springer, Berlin (2008)

16. Fomin, F.V., Kratsch, D., Todinca, I., Villanger, Y.: Exact algorithms for treewidth and minimum fill-in. SIAM J. Comput. 38(3), 1058-1079 (2008)

17. Fortnow, L., Santhanam, R.: Infeasibility of instance compression and succinct PCPs for NP. J. Comput. Syst. Sci. 77(1), 91-106 (2011)

18. Gavril, F.: Some NP-complete problems on graphs. In: 11th Conference on Information Sciences and Systems, pp. 91-95 (1977)

19. Heggernes, P., van't Hof, P., Lokshtanov, D., Nederlof, J.: Computing the cutwidth of bipartite permutation graphs in linear time. In: Thilikos, D.M. (ed.) WG. Lecture Notes in Computer Science, vol. 6410, pp. 75-87 (2010)

20. Heggernes, P., Lokshtanov, D., Mihai, R., Papadopoulos, C.: Cutwidth of split graphs and threshold graphs. SIAM J. Discrete Math. 25(3), 1418-1437 (2011)

21. Held, M., Karp, R.M.: A dynamic programming approach to sequencing problems. J. Soc. Ind. Appl. Math. 10(1), 196-210 (1962)

22. Jansen, B.M.P., Kratsch, S.: Data reduction for graph coloring problems. In: Owe, O., Steffen, M., Telle, J.A. (eds.) FCT. Lecture Notes in Computer Science, vol. 6914, pp. 90-101. Springer, Berlin (2011)

23. Junguer, M., Reinelt, G., Rinaldi, G.: The travelling salesman problem. In: Handbook on Operations Research and Management Sciences, vol. 7, pp. 225-330 (1995)

24. Karger, D.R.: A randomized fully polynomial time approximation scheme for the all-terminal network reliability problem. SIAM J. Comput. 29(2), 492-514 (1999)

25. Karp, R.M.: Dynamic programming meets the principle of inclusion and exclusion. Oper. Res. Lett. 1, 49-51 (1982)

26. Leighton, F., Rao, S.: Multicommodity max-flow min-cut theorems and their use in designing approximation algorithms. J. ACM 46, 787-832 (1999) 
27. Makedon, F., Sudborough, I.H.: On minimizing width in linear layouts. Discrete Appl. Math. 23, 243-265 (1989)

28. Monien, B., Sudborough, I.H.: Min cut is NP-complete for edge weighted trees. Theor. Comput. Sci. 58, 209-229 (1988)

29. Mutzel, P.: A polyhedral approach to planar augmentation and related problems. In: Spirakis, P.G. (ed.) ESA. Lecture Notes in Computer Science, vol. 979, pp. 494-507. Springer, Berlin (1995)

30. Suchan, K., Villanger, Y.: Computing pathwidth faster than $2^{n}$. In: Chen, J., Fomin, F.V. (eds.) IWPEC. Lecture Notes in Computer Science, vol. 5917, pp. 324-335. Springer, Berlin (2009)

31. Thilikos, D.M., Serna, M.J., Bodlaender, H.L.: Cutwidth II: Algorithms for partial w-trees of bounded degree. J. Algorithms 56, 24-49 (2005)

32. Yannakakis, M.: A polynomial algorithm for the min cut linear arrangement of trees. J. ACM 32, 950-988 (1985)

33. Yap, C.K.: Some consequences of non-uniform conditions on uniform classes. Theor. Comput. Sci. 26, 287-300 (1983)

34. Yuan, J., Zhou, S.: Optimal labelling of unit interval graphs. Appl. Math. J. Chin. Univ. Ser. A 10, 337-344 (1995) 